\title{
Design of a Haptic Device for Teleoperation and Virtual Reality Systems
}

\author{
Mehmet İsmet Can Dede, Özgün Selvi, Tunç \\ Bilgincan \\ Department of Mechanical Engineering \\ İzmir Institute of Technology \\ İzmir, Turkey \\ candede@iyte.edu.tr
}

\author{
Yalkın Kant \\ Department of Mechanical Engineering \\ Dokuz Eylül University \\ İzmir, Turkey
}

\begin{abstract}
Haptics technology has increased the precision and telepresence of the teleoperation and precision of the in-house robotic applications by force and surface information feedback. Force feedback is achieved through sending back the pressure and force information via a haptic device as the information is created or measured at the point of interest. In order to configure such a system, design, analysis and production processes of a haptic device, which is suitable for that specific application, becomes important. Today, haptic devices find use in assistive surgical robotics and most of the teleoperation systems. These devices are also extensively utilized in simulators to train medical and military personnel. The objective of this work is to design a haptic device with a new structure that has the potential to increase the precision of the robotic operation. Thus, literature is reviewed and possible robot manipulator designs are investigated to increase the precision in haptics applications. As a result of the investigations, conceptual designs are developed. Ultimately, final design is selected and produced after it is investigated in computer-aided-design (CAD) environment and its kinematic and structural analyses are carried out.
\end{abstract}

Keywords-haptics, robot design, parallel platfom manipulator, hybrid manipulator

\section{INTRODUCTION}

The sense of touch is a viable feedback to understand the environment. Most of the time, the sense of touch accompanied with the visual feedback is enough to gather most of the information about a certain environment. This information is used by robotic interfaces to pass the environment data to the human operator in haptic devices. Haptic systems have been utilized in various state-of-the-art applications, such as teleoperation systems, computer-aided-design (CAD), assistive robotic surgery, telesurgery, computer-aided trainings of the military personnel and surgeons. In teleoperation and telesurgery applications, telepresence is enhanced through haptic devices that feed back force. In training or simulator systems, the force information is used along with the virtual reality information to precisely simulate a real world operation. Haptic devices employed in assistive robotic surgeries provide a precise surgical procedure that will improve and shorten the healing process. Free-form modeling is possible with the use of haptic devices in CAD.
Although serial haptic manipulators have been used in a wide variety of applications and have large workspaces, their relatively low stiffness and low force feedback capacity are their weak sides. Probably the most well known serial haptic device is the Phantom Haptic Device [1]. In order to increase stiffness and force feedback capacity, parallel platform haptic manipulators have several applications. Joon-Woo Kim et al. designed Delta type parallel haptic device with one redundant actuator [2]. Masaru Uchiyama et al. developed a new six degree-of-freedom (DoF) haptic interface. In their work [3], they used a novel spatial gimbal five bar mechanism for orientation and delta manipulator for positioning.

Haptics have many applications for general purposes. M. Muldera et al. introduced haptic gas pedal for automation sector [4]. The test results were promising that the haptic feedback was presented to the drivers, car-following performance was improved. Hong Gee Sim et al. [5] emphasized the necessity of haptic feedback in surgical operations and the haptic da Vinci robotic surgical system, as presented in their work. Haptics is also a solution to nanometer scale operations. Haptics technologies introduced and applied to nanotechnology applications in the $[6,7]$.

Haptic devices have also found use in medical training applications that are accompanied with virtual reality information. Payandeh and $\mathrm{Li}$ [8], have investigated the application of a low-cost electromechanic haptic technology in virtual laparoscopic surgeries. Another virtual reality application for training purposes integrates the force-feedback information from a needle with the virtual world in [9]. P. Wang et al. [10], have introduced a virtual reality device that produces virtual tissue through boundary element method.

In this work, with its high precision, robustness, stiffness, higher load carrying capacity and with three actuators located at the base, a new type of six degree-of-freedom (DoF) hybrid haptic device is introduced. It is composed of the R-Cube structure for translational motions and Hybrid-Spherical structure for the orientating the end-effector. The structural design of the device was selected after examining several conceptual designs in CAD software environment. In the next section, background information is provided on the existing manipulator types and previously developed haptic devices. 
Later, conceptual designs are explained. Then, final design selection and its various analyses are described. Finally, constructed first prototype is shown along with the future work for improving the design.

\section{BACKGROUND}

As a first step before forming the conceptual designs, literature is investigated for suitable manipulators and for their advantages and disadvantages. Basically three types of manipulators can be used in designing of haptic devices; Serial manipulators, Parallel manipulators and Hybrid manipulators.

Serial manipulators are the most common industrial robots. They often have an anthropomorphic mechanical arm structure, which is a serial chain of links, connected by joints, forming a shoulder, an elbow, and a wrist. Their main advantage is their larger workspace with respect to their own volume and occupied floor space. Their main disadvantage is the low stiffness inherent to an open kinematic structure. Also, position errors are accumulated from link to link.

A parallel manipulator can be defined as a closed loop mechanism with an end-effector also called as platform. In parallel manipulators, the platform of the end effector must be connected to the base at least two independent kinematic chains where each chain contains one actuator at minimum. Parallel manipulators have some advantages with respect to serial manipulators such as higher precision, robustness, stiffness, and load carrying capacity. The actuators can be placed in the base platform, so that their mass does not have to be moved, which makes the construction lighter and decrease the inertia of the moving parts. Their major drawback is their limited workspace and low stiffness in singular positions.

Hybrid manipulator can be simply defined by the combination of parallel and serial manipulators. Parallel parallel or parallel - serial constructions can be used. They have the advantages of serial and parallel manipulators and the motions can be designed in decoupled fashion.

There are many manipulators in literature that can meet specifications fro this work. For narrowing down the research, decoupled manipulators are investigated where three actuators controls translational motion and the rest of the actuators monitor orientation which is appropriate for our task.

There are three types of coupling. Strong coupling means that each pose parameter is a function of all actuated joint variables. In complete decoupling, each pose parameter is a function of only one actuated joint variable. Whereas in partial decoupling, partial pose parameters such as translation and rotation are functions of separate actuated joint variables.

Partial decoupling is found in serial arms. Where translation is obtained by first three actuators and orientation is controlled by last three actuators. The advantage of using this type of serial manipulators is the ease in the construction process and no recess joint is used as it is the case with parallel platform manipulators. All joints are actuated, but this advantage converts to a disadvantage in precision specifications because the error of each joint is accumulated.
Advantages of the parallel manipulators are that the motions are partially decoupled, actuators are near to the base and precision lost are decreased because position errors of the actuators are not added but shared. Disadvantage is that these are hard to construct with lots of joints and links and their work spaces are relatively smaller.

Hybrid manipulators are all self-decoupled. Using different manipulators for translation and orientation will introduce many advantages of the serial and parallel manipulators.

For the translation parallel manipulators will be better because of motors are on the base. Many Cartesian robots are available. Some of them can be listed as Delta, Star, Orhoglide [11] and R-Cube [12]. The distinct manipulator in here is the $\mathrm{R}$-Cube because of decoupled motion and use of revolute joints only. However, Delta robot is a common Cartesian robot mostly used in haptic devices.

The orientation of the end-effector can be monitored by serial-spherical, hybrid-spherical or Agile Eye [13] manipulator. Serial manipulator has the maximum workspace and hybrid-spherical manipulator has easier kinematic calculations with respect to others.

\section{CONCEPTUAL Designs}

In the literature survey, possible designs for configuring a high precision haptic device are investigated. As a result of the literature survey, it is decided to achieve translational motion and orientation motion separately through a hybrid structure for the robot. Therefore, the haptic device design considered in this work is developed as two separate parts as the main body and gripping arm. Delta, Cartesian Parallel, and R-Cube manipulators are depicted for translational motion (main body) and for orienting the end-effector (gripping arm), Agile Eye, Hybrid-Spherical and Serial-Spherical manipulators are selected.

\section{A. Delta Manipulator}

Delta robot is a 3DoF-3RRNR parallel manipulator as shown in Fig. 1. Only three joints are actuated. It has coupled motion with grounded rotary actuators. It has seventeen link connected with twenty-one joints, It is easy to solve its inverse and direct kinematic analysis. Its specifications are high resolution, easy control, availability of stability under big forces (high load capacity), fast cycle time with high precision with relatively limited workspace.

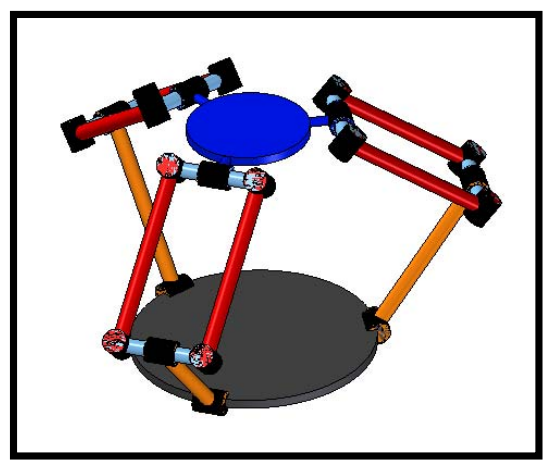

Figure 1. Delta Manipulator 


\section{B. Cartesian Parallel Manipulator}

Cartesian Manipulator is a 3DoF-3PRRR manipulator as shown in Fig. 2. It has decoupled motion with grounded prismatic actuators. It has eleven links connected with twelve joints. It is relatively easier to solve for its inverse and direct kinematic analysis.

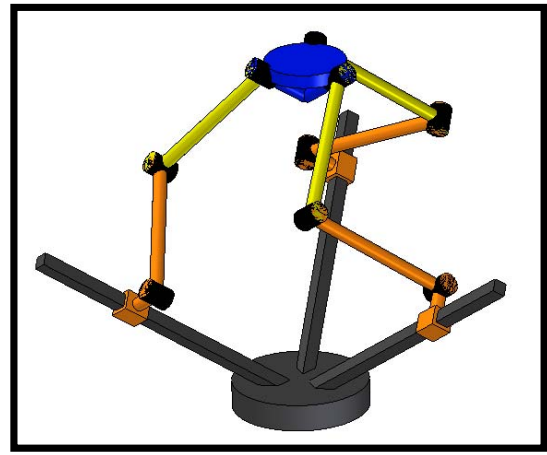

Figure 2. Cartesian Parallel Manipulator

\section{R-Cube Manipulator}

R-Cube Manipulator is a $3 \mathrm{DoF}-3 \mathrm{NRRR}$ manipulator as shown in Fig. 3. It has decoupled motion with rotary actuators placed on the ground frame. It has seventeen links connected with twenty one joints. It is relatively easy to solve for its inverse and direct kinematic analysis [12].

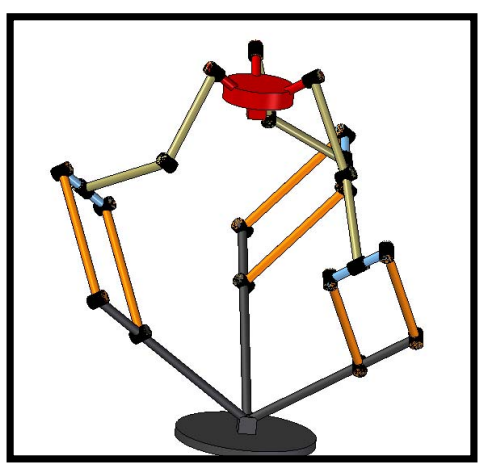

Figure 3. R-Cube Manipulator

\section{Agile Eye}

Agile Eye is a 3DoF-3RRR parallel manipulator with coupled motion as shown in Fig. 4. It has eight links connected with nine joints; because of its construction advantage it has grounded sensors. , It is easy to solve for its inverse kinematic analysis. Its workspace is a cone with $140^{\circ}$ with $\pm 30^{\circ}$ in torsion [13].

\section{E. Hybrid-Spherical Manipulator}

Hybrid-Spherical is a $3 \mathrm{DoF}-2 \mathrm{RE}+\mathrm{R}$ Hybrid type manipulator with decoupled motion as shown in Fig. 2(b). It has four links connected with six joints, related to its structure it has 2 grounded and 1 floating sensor. It's inverse kinematic analysis easy to solve. It has a workspace of $\pm 90^{\circ}(\mathrm{x}) \pm 90^{\circ}(\mathrm{y})$ $\pm 120^{\circ}$ (end effector axis).

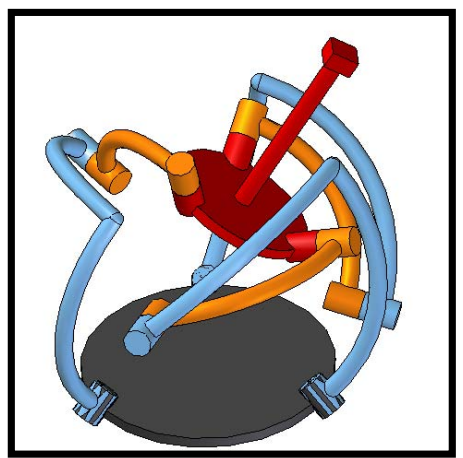

Figure 4. Agile Eye

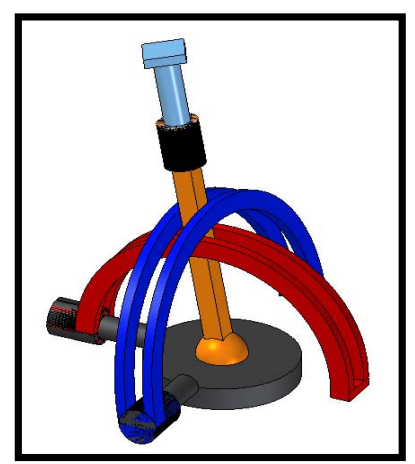

Figure 5. Hybrid-Spherical Manipulator

\section{F. Serial-Spherical Manipulator}

Serial Spherical Manipulator is a 3DoF-RRR manipulator with coupled motion as shown in Fig. 2(c). It has four links connected with six joints. Its inverse kinematic analysis is complex and its workspace is $\pm 120^{\circ}$ around all directions.

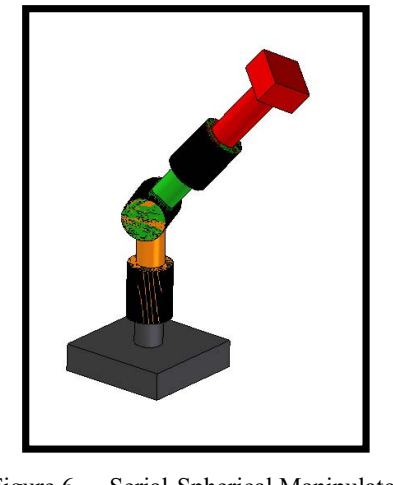

Figure 6. Serial-Spherical Manipulator

\section{FINAL DESIGN}

The conceptual designs for the two main mechanisms of the haptic device are discussed and investigated in the Tables I and II. Among the main body candidates, R-cube includes only revolute joints and it is relatively simpler to manufacture both revolute joints and the structure of this mechanism. The decoupled motion of the R-cube and the Hybrid-Spherical manipulators results in relatively easy direct and inverse kinematic calculations. Thus, Hybrid-Spherical and R-cube 
mechanisms are selected to configure the haptic device. The selected mechanism has a different structure than the commercially available systems by Force Dimension [15]. They use a Delta-based structure in their systems.

The production of the final design prototype has two stages as design of the mechanism and the controller. Structural and kinematic analyses are carried out for the mechanism as it is presented in the next subsections. Later, in CAD environment, mechanism design is finalized and further analysis is carried out for estimated actuator torques.

TABLE I. PRoperties of THE CONCEPTUAL Main Body Designs

\begin{tabular}{|c|c|c|c|}
\hline & Delta & Cartesian & R-Cube \\
\hline \# of Joints & 21 & 12 & 21 \\
\hline \# of Links & 17 & 11 & 17 \\
\hline Kinematic Analysis & Complex & Simple & Simple \\
\hline Uncoupled Motion & No & Yes & Yes \\
\hline Actuation & Rotary & Translational & Rotary \\
\hline Grounded Actuators & Yes & Yes & Yes \\
\hline Joint Types & R & R-P & R \\
\hline Cost & Low & Medium & Low \\
\hline
\end{tabular}

TABLE II. PROPERTIES OF THE CONCEPTUAL GRIPPING ARM DESIGNS

\begin{tabular}{|c|c|c|c|}
\hline & Agile Eye & $\begin{array}{c}\text { Hybrid- } \\
\text { Spherical }\end{array}$ & $\begin{array}{c}\text { Serial- } \\
\text { Spherical }\end{array}$ \\
\hline \# of Joints & 9 & 6 & 3 \\
\hline \# of Links & 8 & 4 & 4 \\
\hline $\begin{array}{c}\text { Kinematic } \\
\text { Analysis }\end{array}$ & Simple & $\begin{array}{c}\text { Partially } \\
\text { complex }\end{array}$ & Complex \\
\hline $\begin{array}{c}\text { Uncoupled } \\
\text { Motion }\end{array}$ & No & Yes & No \\
\hline $\begin{array}{c}\text { Actuation } \\
\text { Cone } 140^{\circ} \\
\text { torsion } \pm 30^{\circ}\end{array}$ & $\begin{array}{c} \pm 90^{\circ} \pm 90^{\circ} \\
\pm 120^{\circ}\end{array}$ & $\begin{array}{c} \pm 120^{\circ} \pm 120^{\circ} \\
\pm 120^{\circ}\end{array}$ \\
\hline $\begin{array}{c}\text { Grounded } \\
\text { Actuators }\end{array}$ & Yes & Partially & No \\
\hline $\begin{array}{c}\text { Joint } \\
\text { Types }\end{array}$ & $\mathrm{R}$ & R-S-E & $\mathrm{R}$ \\
\hline Cost & High & Medium & Low \\
\hline
\end{tabular}

The links of the haptic device is manufactured from stainless steel with laser cutting. Stainless steel is selected for its resistance to corrosion and heavy loads. For the first prototype, sliding joint architecture is selected for its low cost and assembly easiness even though it decreases the precision. Hybrid spherical mechanism from a manual joystick was used as the gripping arm.

A custom electronic card is designed and connected by opto-couplers against possible circuit faults. Card has two power supplies $5 \mathrm{~V}-1 \mathrm{~A}$ and $5 \mathrm{~V} 20 \mathrm{~A}$ to overcome the fluctuation of the voltage. Pic16f877 is chosen as the microcontroller due to its capability of handling both digital and analog signals. Three stepper motors are implemented for actuation and for the location feedback, potentiometers are used.

The coordinates of the manipulator was changed to have the coordinate systems of the actuator parallel to the ground frame in order to maximize the workspace (Fig. 7). The link lengths are then optimized and tested against for collision in CAD.

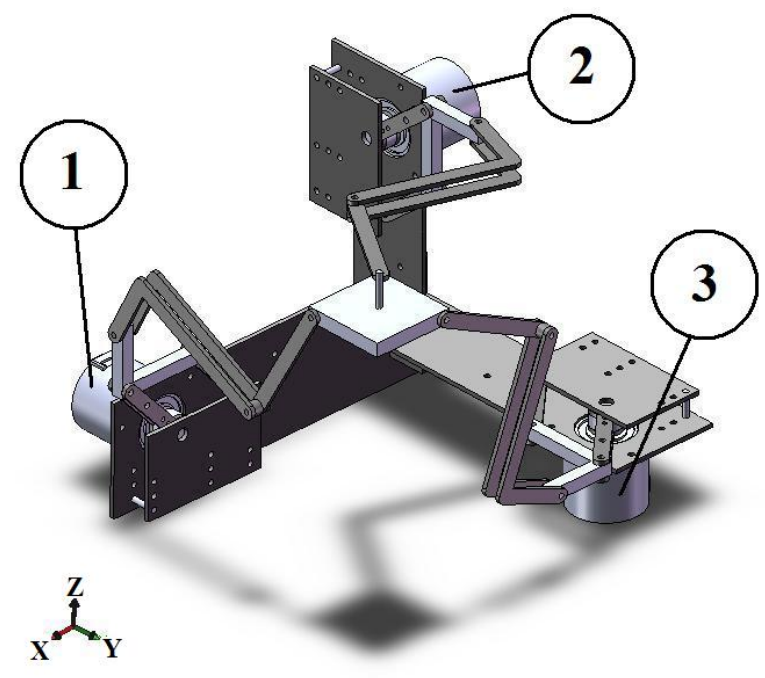

Figure 7. Final Design

\section{A. Strutural Analysis}

Main body of the robot is a three DoF parallel platform manipulator. The structural properties of the manipulator in Table III are provided using the notation given in [14]. In this table; $\lambda$ is the degree of freedom of an unconstrained rigid body moving in the workspace of the mechanism, $B$ is the number of mobile platforms, $j_{p}$ is the total number of joints on the mobile platforms, $\mathrm{c}_{\mathrm{L}}$ is the total number of legs of the manipulator, $L$ is the number of independent loops, $f_{T}$ is the sum of mobility of all joints in the structural group.

TABLE III. Structural Properties OF THE MANIPUlator

\begin{tabular}{|c|c|c|c|c|c|}
\hline$\lambda$ & $\boldsymbol{B}$ & $\boldsymbol{j}_{\boldsymbol{p}}$ & $\boldsymbol{c}_{\boldsymbol{L}}$ & $\boldsymbol{L}$ & $\boldsymbol{f}_{\boldsymbol{T}}$ \\
\hline 6 & 1 & 3 & 3 & 2 & 15 \\
\hline Class & Kind & Type & Order & \multicolumn{2}{|c|}{ Modification } \\
\hline 1 & 0 & 3 & 3 & \multicolumn{2}{|c|}{1} \\
\hline
\end{tabular}

The DoF of the manipulator can be calculated using (1) as $M=(1-3) 6+15=3$ with the properties provided in Table III.

$$
M=(B-C) \lambda+\sum f_{i}
$$

In this equation; $\Sigma f i$ is the total number of joints, $C$ is the total number of legs, and $B$ is the total number of moving platforms.

\section{B. Kinematic Analysis}

In this structure, positions of the platform are determined by the positions of the actuators. As the manipulator is an uncoupled one, motion in each axes $\{\mathrm{x}, \mathrm{y}, \mathrm{z}\}$ will be dependent on only one actuator in each leg. 
The direct and inverse kinematic analysis of the manipulator is relatively easier due to the fact that the motion of the manipulator in each axis is uncoupled.

Fig. 8 shows a coordinate relationship for the kinematic analysis of the parallel structure. Base frame $\{\mathrm{O}\}$ and the moving frame $\{\mathrm{E}\}$ of end-effector is located as shown in Fig. 8. Note that vector $p$ represents the changes in the position of moving frame $\{\mathrm{E}\}$ with respect to the fixed frame $\{\mathrm{O}\}$. The parameters $h_{i}$ and $l_{i}$ are the distance of ground joint to ground frame $\{\mathrm{O}\}$ coordinate center and the length of the actuated link respectively.

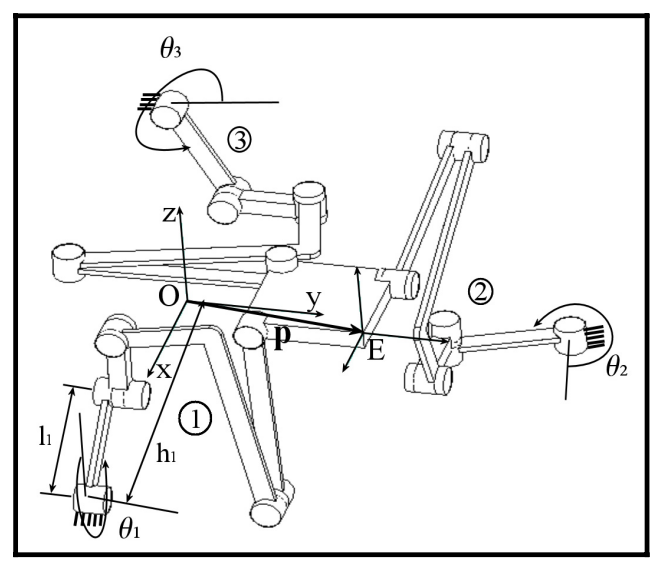

Figure 8. Analysis of the Final Design

Vector $p$ can be represented in terms of the unit vectors of the ground frame as shown in (2).

$$
\vec{p}=P_{1} \vec{u}_{1}+P_{2} \vec{u}_{2}+P_{3} \vec{u}_{3}
$$

Forward kinematic solution is presented in (3).

$$
P_{i}=h_{i}+l_{i} \sin \left(\theta_{i}\right) ; \quad i=1,2,3
$$

The velocity components of the platform is calculated as shown in (4).

$$
V_{i}=l_{i} \cos \left(\theta_{i}\right) \dot{\theta}_{i} ; \quad i=1,2,3
$$

The inverse kinematic solution of the manipulator is given in (5). Using this equation, it is possible to calculate the required positon of the actuators for a desired end-effector position.

$$
\begin{gathered}
\sin \left(\theta_{i}\right)=\frac{P_{i}-h_{i}}{l_{i}} \quad i=1,2,3 \\
\cos \left(\theta_{i}\right)=\sigma \sqrt{1-\sin ^{2}\left(\theta_{i}\right)} ; \quad \sigma= \pm 1 \\
\theta_{i}=\arctan _{2}\left(\sin \left(\theta_{i}\right), \cos \left(\theta_{i}\right)\right)
\end{gathered}
$$


constant gravitational loading. It is not sharing this load with the other actuator as expected from a parallel platform manipulator due to its changed orientation to increase the workspace.

\section{CONCLUSION}

Force and surface information feedback is required for various robotic applications to increase precision and telepresence. Haptic devices are increasingly utilized for this purpose in industrial applications and scientific studies. Mechanism and controller designs and production are important in these systems in which precision is vital.

In this work, it is aimed to configure a new robot structure to be used in haptic systems that will introduce some improvements. In that respect, direct drive joints are used, mechanisms that enable uncoupled motion are selected and RCube mechanism is twisted to enlarge the workspace (Fig. 12). However, the CAD analysis indicates that the change in the orientation of the R-Cube results in higher loading in one of the actuators of the system. Thus, for the second prototype, R-Cube mechanism will be constructed in its original orientation. Further investigation on the mechanism will be made during the design and construction phase of the second prototype.

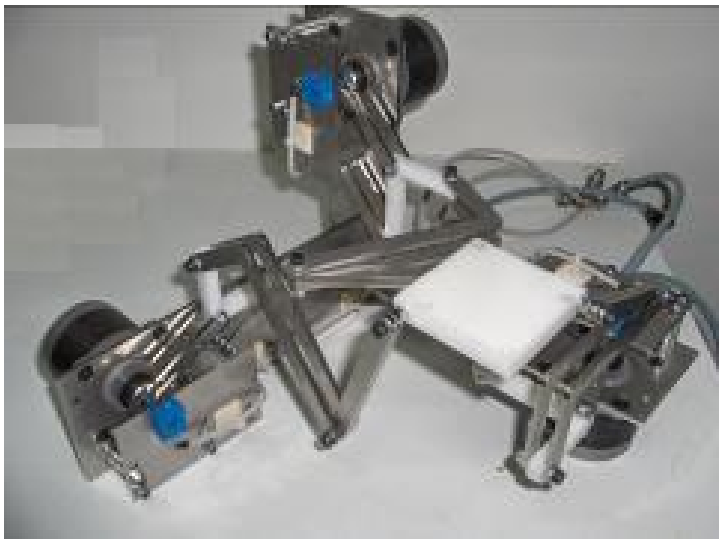

Figure 12. First Working Prototype

It is planned to use bearings instead of sliding joints to increase the precision. Also, it is necessary to change the stepper motors used in the first prototype with powerful servomotors to enable the system to be used as a haptic device. System will be examined in a virtual reality simulator after the planned modifications and the hybrid architecture are achieved.

\section{REFERENCES}

[1] O. Thomas H. Massie and J. K. Salisbury, "The PHANTOM Haptic Interface: A Device for Probing Virtual", American Society of Mechanical Engineers, Dynamic Systems and Control Division DSC, Vol. 55-1, pp. 295-299. Nov. 1994

[2] Joon-Woo Kim, Duk-Hee Park, Han-Sung Kim, Sung-Hyun Han, "Design of a Novel 3-DOF Parallel-type Haptic Device with Redundant Actuation", Control, Automation and Systems, Oct. 2007, 2270-2273.

[3] Masaru Uchiyama, Yuichi Tsumaki, and Woo-Keun Yoon, "Design of a Compact 6-DOF Haptic Device to Use Parallel Mechanisms", Springer Tracks in Advanced Robotics, Springer Berlin / Heidelberg, Volume 28, 2007, 145-162.

[4] M. Muldera, M.M. Van Paassena and D.A. Abbink, "Haptic gas pedal feedback", Ergonomics, vol 51 (issue 11),2008-Nov, pp 1710-1720.

[5] Hong Gee Sim, Sidney Kam Hung Yip, Christopher Wai Sam Cheng, "Equipment and technology in surgical robotics", World Journal of Urology, Springer Berlin / Heidelberg, Volume 24, Number 2, June, 2006, pp. 128-135.

[6] F.J. Rubio-Sierra, R.W. Stark, S. Thalhammer, W.M. Heckl, "Forcefeedback joystick as a low-cost haptic interface for an atomic-forcemicroscopy nanomanipulator", Applied Physics A: Materials Science \& Processing, Springer Berlin / Heidelberg, Volume 76, Number 6, April 2003, pp. 903-906.

[7] Marc Jobin, Raphael Foschia, Sébastien Grange, Charles Baur, Gérard Gremaud, Kyumin Lee, Laszlo Forró, and Andrzej Kulik, "Versatile force-feedback manipulator for nanotechnology applications", Review of Scientific Instruments, Volume 76 Issue 5, 2005.

[8] Shahram Payandeh, Temei Li, "Toward new designs of haptic devices for minimally invasive surgery", International Congress Series Computer Assisted Radiology and Surgery. Proceedings of the 17th International Congress and Exhibition, June 2003, 775-781.

[9] Franck P. Vidala, Nicholas Chalmers, Derek A. Gould, Andrew E. Healey, Nigel W. Johna, "Developing a needle guidance virtual environment with patient-specific data and force feedback", International Congress Series Computer Assisted Radiology and Surgery, May 2005, 418-423.

[10] P. Wang, A.A. Becker, I.A. Jones, A.T. Glover, S.D. Benford, C.M Greenhalgh, M. Vloeberghs, "A virtual reality surgery simulation of cutting and retraction in neurosurgery with force-feedback", Computer methods and programs in biomedicine, vol. 84, 2006, 11-18.

[11] A. Pashkevich, P. Wenger, D. Chablat, "Design strategies for the geometric synthesis of Orthoglide-type mechanisms", Mechanism and machine theory, Elsevier, Oxford, vol. 40, ROYAUME-UNI, 2005, pp. 907-930.

[12] Weimin Li, Feng Gao, Jianjun Zhang, "R-CUBE, a decoupled parallel manipulator only with revolute joints", Mechanism and Machine Theory, Elsevier, Volume 40, Issue 4, April 2005, pp. 467-473.

[13] Gosselin CM, StPierre E, Gagne M, "On the development of the Agile Eye", Robotics \& Automation Magazine, IEEE, vol. 3, Issue 4, Dec 1996, pp. 29-37.

[14] Rasim I Alizade, Çăgdaș Bayram, Structural Synthesis of parallel manipulators, IFToMM J. Mech. Mach. Theory, Vol. 39, 2004, 857-870.

[15] Force Dimension. Retrieved June 20, 2009 from http://www.forcedimension.com. 